\title{
Impacto de dos estrategias educativas en la adquisición de conocimientos sobre diabetes mellitus en adolescentes no diabéticos
}

DOI: $\underline{\text { https://doi.org/10.32870/dse.v0i8.311 }}$

$$
\begin{aligned}
& \text { Rafael Bustos Saldaña*, Blanca Nayeli Aceves González** } \\
& \text { Bertha Alicia del Toro Anay***, Patricia Martínez Har**** } \\
& \text { Federico Pérez Martínez*****, Miguel Gonzalo Rodríguez Riveros****** José } \\
& \text { Miguel Rolón Rodríguez } * * * * * * * \text {,José de Jesús Vargas Quezada******** y } \\
& \text { Noelia del Rocío Vargas Quezada } * * * * * * * * *
\end{aligned}
$$

\begin{abstract}
Resumen: Evaluar dos estrategias educativas en la adquisición de conocimientos sobre diabetes mellitus en adolescentes no diabéticos. MATERIALY MÉTODOS: Diseño: cuasiexperimental. Universo y Muestra: 24 participantes adolescentes divididos en dos grupos de manipulación. Técnicas de aprendizaje utilizadas: a) reforzamiento de preguntas durante el juego y b) realización de actividades de periodismo. Criterios de selección: adolescentes, que voluntariamente aceptaron participar y que no fueran diabéticos. Variables de estudio: conocimientos básicos de la enfermedad, control de glucemia y prevención de complicaciones. Procedimiento: se realizaron 10 sesiones de charlas de 15 minutos sobre diabetes a los dos grupos, el grupo de reforzamiento se le agregó preguntas sobre el tema de la sesión educativa durante su práctica de juego de tenis y al de periodismo se le enseñó a realizar una nota periodística sobre el tema. Se les realizó evaluaciones con el instrumento DKQ24. RESULTADOS: Edad en el grupo de reforzamiento: $12.17 \pm 1.95$ años y en periodismo: $12.25 \pm 1.48$ años, en el grupo de periodismo fueron 3 mujeres y en el de reforzamiento 5 . Los participantes de reforzamiento presentaron $10.5 \pm 2.9$ y $17.92 \pm 3.4(p<0.05)$ respuestas adecuadas al inicio y final del estudio respectivamente, el de periodismo $11 \pm 4.04$ y $14.33 \pm 3.3(p<0.05)$ respectivamente. Las diferencias encontradas al inicio y final en el grupo de reforzamiento fue $7.42 \pm 2.5$ y en el de periodismo $3.33 \pm 3.3(p<0.05)$ respuestas adecuadas.

CONCLUSIÓN: Las dos estrategias educativas mejoraron en la adquisición de conocimientos en diabetes, sin embargo, los participantes del grupo con reforzamiento con preguntas tuvieron mayor aprendizaje. Palabras claves: diabetes mellitus, conocimientos, estrategias educativas.
\end{abstract}

\begin{abstract}
To evaluate two educational strategies in the acquisition of knowledge about diabetes mellitus in non-diabetic adolescents. MATERIAL AND METHODS: Design: Quasi-experimental. Universe and Sample: 24 participants divided into two group's teenagers handling. Learning techniques used to) Strengthening of questions during the game b) Implementation of journalism. Selection criteria: adolescents, who voluntarily agreed to participate and were not diabetic. Study variables: Basic knowledge of the disease, glycemic control and prevention of complications. Procedure: They received 10 sessions of 15 -minute talks on diabetes in the two groups, the group was added reinforcement questions about the educational session topic during tennis practice and game journalism was taught to make a newspaper article on the subject. Evaluation was performed with DKQ24 instrument. RESULTS: Age at strengthening group: $12.17 \pm 1.95$ years and in journalism: $12.25 \pm 1.48$ years. In the journalism group were 3 women and the reinforcement 5. Strengthening participants had $10.5 \pm 2.9$ and $17.92 \pm 3.4(p<0.05)$ appropriate responses at

*Maestro en Ciencias Médicas. Profesor-investigador titular "B". Centro Universitario del Sur de la Universidad de Guadalajara en Ciudad Guzmán, Jalisco. Correo electrónico: rafaelb@cusur.udg.mx, **Licenciada en periodismo, ***Estudiante de medicina, $* * * *$ Estudiante de medicina, $* * * * *$ Licenciado en periodismo, $* * * * * *$ Estudiante de medicina, $* * * * * * *$ Estudiante de medicina, $* * * * * * * *$ Estudiante de medicina, $* * * * * * * * *$ Estudiante de medicina.
\end{abstract}


Rafael Bustos, Blanca Aceves, Bertha del Toro, Patricia Martínez, Federico Pérez, Miguel Rodríguez, José Miguel Rolón, José de Jesús Vargas y Noelia Vargas

the beginning and end of the study, respectively, the journalism $11 \pm 4.04$ and $14.33 \pm 3.3(p<.05)$. The differences found in the beginning and end reinforcement group was $7.42 \pm 2.5$ and $3.33 \pm$ journalism the $3.3(p<0.05)$ suitable answers. CONCLUSION: The two improved educational strategies in the acquisition of knowledge of diabetes, however, the group participants had higher reinforcement learning. Key Words: Diabetes mellitus, Knowledge, educational strategies.

\section{INTRODUCCIÓN}

El mundo está cambiando epidemiológicamente, al aumentar las expectativas de vida en la población se ha incrementado la prevalencia de las enfermedades crónico-degenerativas (Gómez, 2001 \& Kuri, 2011). Actualmente diabetes mellitus (como la mayoría de las enfermedades crónicas degenerativas) se ha convertido en un problema de salud mundial, que probablemente va a tener en corto plazo cambios económicos en los programas de salud asistencial de todos los países (Kuri, 2011 \& Lerman et al., 2009).

La base de la prevención en diabetes debe de ser la educación que se tenga no sólo al paciente, sino también a las familias de ellos y en forma general a la población (Bustos, 2006). Por desgracia esta prevención no ha tenido el impacto esperado y muy poca repercusión en su incidencia y prevalencia (IDF, 2009 y Arredondo, 2011).

La educación en salud en diabetes mellitus ha demostrado beneficios importantes en el diagnóstico, control y tratamiento de los pacientes ya afectados; sin embargo, en la población en general, son pocos los resultados que se han obtenido, principalmente porque este aprendizaje se observará a muy largo plazo (IDF, 2011).

Se ha observado que la educación en salud realizada adecuadamente en los niños y jóvenes en los programas educativos formales en forma general es muy escasa, no obstante, existe evidencia de su impacto en la actitud de los niños ante las enfermedades (Ostergren, et al., 2007).

El niño y el adolescente generalmente presentan una educación en salud solamente como parte agregada de su formación, no como un pilar para su vida futura (UNESCO). Esta educación está considerada para mejorar los estilos de vida saludables en forma general y no a una enfermedad en específico.

Existe un cambio de actitud y conocimientos en los niños y adolescentes cuando se aplican técnicas participativas en el aprendizaje de temas de salud, principalmente en conocimientos, habilidades y actitudes, no sólo para ellos en particular, sino como promotores de salud en la familia y su comunidad (García, González y Aguilar, 2010; Colomer, et al., 2004 \& Pupo y Carvajal, 2009).

Por otra parte, la atención sanitaria a la población infantil constituye actualmente un elemento básico de la Atención Primaria de Salud. En la edad infantil la prevención primaria presenta una gran efectividad, ya que las principales causas de mortalidad se relacionan con hábitos que comienzan a desarrollarse en la infancia y adolescencia o para prevenir hábitos nocivos que puedan tener repercusión en su vida adulta (Aguilera et al., 2005 y Caldes et al., 2005). La adopción de comportamientos saludables es un proceso laborioso, por un lado se debe de tener en cuenta que las personas primeramente necesitan tener informa- 
Impacto de dos estrategias educativas en la adquisición de conocimientos sobre diabetes mellitus

ción sobre qué hacer y cómo hacerlo, pero por otra parte, dichos aspectos entran en contradicción con el conocimiento práctico heredado de generación en generación (Guibert, 1999).

El objetivo del trabajo fue evaluar dos estrategias educativas (una a base de reforzamiento de preguntas en el juego y otra a base de elaboración de un periódico sobre diabetes) en la adquisición de conocimientos sobre diabetes mellitus en adolescentes no diabéticos de 11 a 17 años de edad.

\section{Material y métodos}

Diseño de estudio: cuasiexperimental, donde se parearon los integrantes en dos grupos, de acuerdo a las calificaciones iníciales del examen de conocimientos.

Universo y muestra: 24 adolescentes que llevaron el Taller de Tenis en el Curso de Verano 2013 del Centro Universitario del Sur de la Universidad de Guadalajara en Ciudad Guzmán, Jalisco, México.

Criterios de selección: Adolescentes de 11 a 17 años no diabéticos registrados en el Taller de Tenis, que voluntariamente aceptaron sus padres la intervención de sus hijos y los participantes asintieron en un consentimiento firmado. Para haber sido considerados en el estudio los adolescentes debieron cursar 80 por ciento de las pláticas que se realizaron y contestar la evaluación de conocimientos al inicio y final del estudio.

Variables de estudio: Conocimientos básicos de la enfermedad, control de glucemia y prevención de complicaciones.

Procedimiento: Previa autorización por escrito de los padres y con el respectivo asentimiento de los adolescentes, se les aplicó el instrumento DKQ 24 (Diabetes Knowledge Questionnaire 24) (García et al., 2001) al inicio del trabajo. Este cuestionario tiene una base de 24 reactivos con respuestas establecidas de: Si, No, o No Sé. El total de reactivos se dividieron en 3 secciones (Bustos et al., 2011): a) conocimientos básicos, b) control de la glucemia y c) prevención de complicaciones. Se optó por utilizar este instrumento porque ya había sido validado con anterioridad en personas de la región que cursaron escolaridad primaria, por lo que su aplicación no tuvo inconvenientes de lenguaje.

Procedimiento para contestar el instrumento: Se leyó por parte de los investigadores en forma general las preguntas textualmente ( $\sin$ hacer ningún tipo de comentarios), para que, los adolescentes lo respondieran en forma individual en una hoja de respuestas otorgadas a cada uno de ellos.

Designación de grupos experimentales: Para la designación de los grupos de manipulación se parearon a los adolescentes de acuerdo a las calificaciones obtenidas en el examen inicial, por lo que se establecieron en: 1.- Grupo de Reforzamiento de preguntas en el juego ( $\mathrm{n}=12)$ y 2.- Grupo de Actividades con reforzamiento en Periodismo $(\mathrm{n}=12)$. 
Rafael Bustos, Blanca Aceves, Bertha del Toro, Patricia Martínez, Federico Pérez, Miguel Rodríguez, José Miguel Rolón, José de Jesús Vargas y Noelia Vargas

\section{Desarrollo de las estrategias educativas}

Actividades educativas comunes: Los dos grupos (juntos) tuvieron 10 pláticas-charlas (aproximadamente de 15 minutos cada una de ellas) en un salón acondicionado especialmente, acerca de temas relacionados con diabetes mellitus, como preámbulo de sus actividades del Taller de Tenis.

Las pláticas fueron realizadas de acuerdo a la siguiente temática: A) conocimientos básicos: 1) insulina y metabolismo de la glucosa, 2) definición y tipos de diabetes, 3) factores de riesgo de diabetes mellitus. B) control de la enfermedad: 1) alimentación, 2) ejercicio, 3) medicamentos, 4) instrumentos y control de glucemia y C) prevención de complicaciones: 1) en el sistema nervioso, 2) en los riñones y 3 ) en la circulación sanguínea y cuidados de sus pies.

Las charlas otorgadas fueron realizadas por una sola persona, tomando en cuenta el paradigma participativo del aprendizaje (36). Los temas de las pláticas se basaron primeramente en los conocimientos previos que presentaban los adolescentes, sus habilidades y las actitudes sobre la enfermedad. Con base a lo anterior, se realizaba el contenido de la charla encauzándolo al interés de los participantes y el objetivo de cada una de ellas. El objetivo de cada una de las sesiones era de establecer conocimientos del tema comunes en los participantes, por medio de la reflexión e incorporación a su estructura de pensamiento.

No se utilizó material audiovisual agregado, por lo que, esta actividad estuvo supeditada principalmente a la atención de los adolescentes y su entendimiento.

Al término de cada una de las charlas los participantes asistieron al Taller de Tenis a realizar sus actividades deportivas.

Actividades comunes deportivas: en conjunto los dos grupos presentaban un calentamiento y estiramiento físico de aproximadamente 10 minutos de duración. Posterior a esto se dividía el taller en los grupos de trabajo para realizar lo siguiente:

\section{a) Grupo de reforzamiento con preguntas durante la actividad física:}

Preparación de material de reforzamiento: Previamente a la realización de cada uno de los temas, los investigadores realizaron 15 preguntas "abiertas" y reflexivas sobre el contenido de las pláticas para las actividades de reforzamiento (independientes del instrumento utilizado -DKQ24-), por lo que en total se tuvieron 150 reactivos.

1.- Actividades deportivas: Se les explicó la técnica para realizar las distintas jugadas de tenis, posterior a esto se dividían en dos grupos para realizar su práctica durante aproximadamente 30 minutos.

2.- Actividades de reforzamiento educativo: Durante la realización de su práctica de tenis, se les realizaba en forma individual a cada uno de los participantes (reforzamiento individual) las preguntas que se habían elaborado con anterioridad sobre el tema que se trató en la charla (una por cada ocasión que tenían actividad con su raqueta y pelotas). 
Impacto de dos estrategias educativas en la adquisición de conocimientos sobre diabetes mellitus

Se efectuó una competencia entre los dos subgrupos formados para obtener ganadores, esto propició en los participantes que trataran de contestar en forma adecuada y rápida para ganarle al equipo contrario. Usualmente cada adolescente tuvo que contestar alrededor de 10 a 15 preguntas en cada una de sus sesiones deportivas.

Al término de la práctica de tenis los participantes pasaban a un área de acondicionamiento físico por un total de 30 minutos, para practicar ejercicios aeróbicos de acuerdo a su edad. Durante cada una de las rutinas que realizaron se les preguntó en forma grupal (reforzamiento en grupo) los mismos cuestionamientos que se habían realizado anteriormente en forma individual.

\section{b) Grupo de manipulación con reforzamiento en el Periodismo:}

1.- Actividades deportivas: Iniciaron con la realización de actividades de acondicionamiento físico de acuerdo a su edad por 30 minutos y posteriormente efectuaron las actividades de enseñanza de técnica de tenis y su juego por otros 30 minutos más (esta actividad fue similar a la realizada en el Grupo de Reforzamiento de preguntas).

2.- Actividades de reforzamiento educativo: Durante las 6 primeras sesiones (charlas) no presentaron actividades de reforzamiento de aprendizaje de conocimientos en diabetes mellitus. A partir de la $7^{\mathrm{a}}$ sesión tuvieron un curso adicionado sobre periodismo y la forma de elaborar notas periodísticas. Los temas que se tuvieron en estas sesiones fueron: a) el periódico y sus partes componentes, b) la elaboración de una nota periodística c) las preguntas que contesta un periodista al efectuar una nota y d) la realización de una nota periodística con respecto a diabetes. En el último día de sesiones ( $10^{\circ}$ día) los adolescentes realizaron un periódico (con titulares, notas cortas, editoriales y entrevistas) de 4 hojas sobre diabetes mellitus, en esta actividad los alumnos (sin reforzamiento por los asesores de elaboración del periódico) recordaron y plasmaron en su proyecto final los conocimientos adquiridos durante el curso.

Al día siguiente de la $10^{\mathrm{a}}$ charla con los dos grupos se procedió a efectuar la aplicación del cuestionario DKQ 24 de la misma forma que se realizó al inicio del estudio. (Esquema metodológico del estudio).

Análisis estadístico: Los resultados de edad, escolaridad, total de conocimientos, conocimientos básicos, control de glucemia y prevención de complicaciones se realizaron a través de media \pm desviación estándar, para la descripción de sexo, familiares diabéticos y la respuesta adecuada de cada una de los ítems del cuestionario se llevó a cabo con frecuencia simple y porcentaje.

Para la comparación de los promedios entre los dos grupos primeramente se utilizó el test de Bartlet para valorar la normalidad de las distribuciones (todas las veces que se realizó arrojó $\mathrm{p}>0.05$ ), posterior a lo cual se aplicó t de Student para muestras no pareadas en el momento de comparar los basales de cada uno de los grupos y t de Student pareada para comparar los resultados del inicio y final de cada grupo. En el caso de la comparación de las frecuencias, se utilizó Chi 
Rafael Bustos, Blanca Aceves, Bertha del Toro, Patricia Martínez, Federico Pérez, Miguel Rodríguez, José Miguel Rolón, José de Jesús Vargas y Noelia Vargas

cuadrada con corrección de Yates y test exacto de Fisher. Se tomó una significancia estadística de p $<0.05$. Se utilizó la ayuda de los programas estadísticos de EPI INFO 2000 y SIGMA STAT 3.1.

Aspectos éticos: El presente estudio fue aceptado por el Colegio Departamental de Salud y Bienestar del Centro Universitario del Sur de la Universidad de Guadalajara de Ciudad Guzmán, Jalisco, México. En todos los participantes se utilizó un consentimiento informado con autorización de sus padres y asentimiento de los adolescentes. Se mantuvieron los preceptos indicados en la Convención de Helsinki y sus enmiendas posteriores para los estudios experimentales en seres humanos.

\section{Resultados}

Características generales: la edad de los participantes del grupo de reforzamiento con preguntas en el juego fue de $12.17 \pm 1.95$ años y en los de periodismo $12.25 \pm 1.48$ años. En cuanto al género de los participantes en el grupo de reforzamiento fueron 5 mujeres y 7 hombres, mientas que en el de periodismo se encontraron 3 mujeres y 9 hombres. En el aspecto de escolaridad el grupo de reforzamiento presento $7.25 \pm 1.71$ años y el de periodismo $7 \pm 2.04$ años de estudio. Por último, el grupo de reforzamiento presentó 5 familiares diabéticos en comparación de 10 en el de periodismo.

\section{Respuestas al cuestionario $\mathrm{DKO}_{24}$}

Características generales: al inicio del estudio los adolescentes presentaban conocimientos adecuados sobre la enfermedad principalmente en los rubros de: el papel de los alimentos y la insulina, la importancia de preparar los alimentos, valores altos de glucemia y las complicaciones a largo plazo de diabetes.

Al final del estudio los participantes mejoraron en general en muchos rubros como son: la causa de la enfermedad, herencia, curación, tipos, y mejoraron sustancialmente en los conocimientos sobre las complicaciones de la enfermedad.

La tabla 1 muestra las respuestas adecuadas realizadas para cada uno de los ítems que se desarrollaron en el cuestionario.

Diferencias entre los grupos: Los promedios de conocimiento de las respuestas del cuestionario fueron semejantes al inicio, todos ellos fueron menores a 50 por ciento de las posibles contestaciones adecuadas. El grupo de reforzamiento a base de preguntas, tuvo cambios significativos positivos en todos los rubros de clasificación de preguntas. El grupo con reforzamiento de periodismo presento mejoría solamente en prevención de complicaciones de la enfermedad.

Los participantes del grupo de reforzamiento con preguntas presentaron mejoría en lo referente a conocimientos básicos y control de glucemia que el grupo de reforzamiento con periodismo.

En forma general los adolescentes pertenecientes al grupo de reforzamiento con preguntas, presentaron un cambio significativo mayor en su conocimiento que aquellos del grupo de reforzamiento de Periodismo. La tabla 2 muestra la diferencia de promedios que obtuvieron los alumnos de acuerdo a las respuestas adecuadas emitidas. 
Impacto de dos estrategias educativas en la adquisición de conocimientos sobre diabetes mellitus

Tabla 1: Respuestas adecuadas al cuestionario DKQ 24 de acuerdo a cada uno de los grupos de estudio

\begin{tabular}{|c|c|c|c|c|c|c|}
\hline \multirow[b]{2}{*}{ CONOCIMIENTOS BASICOS } & \multicolumn{3}{|c|}{ GRUPO REFORZAMIENTO } & \multicolumn{3}{|c|}{ GRUPO DE PERIODISMO } \\
\hline & $\begin{array}{l}\text { RESPUESTA } \\
\text { INICIAL }\end{array}$ & $\begin{array}{l}\text { RESPUESTA } \\
\text { FINAL }\end{array}$ & $\begin{array}{l}\text { DIFERENCIA } \\
\text { ENTRE RES- } \\
\text { PUESTAS }\end{array}$ & $\begin{array}{l}\text { RES- } \\
\text { PUESTA } \\
\text { INICIAL }\end{array}$ & \begin{tabular}{|l} 
RES- \\
PUESTA \\
FINAL
\end{tabular} & $\begin{array}{l}\text { DIFERENCIA } \\
\text { ENTRE RES- } \\
\text { PUESTAS }\end{array}$ \\
\hline $\begin{array}{l}\text { El comer cosas dulces no es causa de } \\
\text { diabetes }\end{array}$ & 2 & 5 & 3 & 1 & 2 & 1 \\
\hline $\begin{array}{l}\text { La falta de insulina es la causa de dia- } \\
\text { betes }\end{array}$ & 7 & 11 & 4 & 8 & 7 & -1 \\
\hline $\begin{array}{l}\text { Los riñones no causan diabetes por no } \\
\text { poder controlar la glucosa en sangre }\end{array}$ & 6 & 6 & 0 & 6 & 2 & -4 \\
\hline Los riñones no producen insulina & 3 & 5 & 2 & 7 & 6 & -1 \\
\hline $\begin{array}{l}\text { Si soy diabético, mis hijos tendrán más } \\
\text { riesgo de padecerla }\end{array}$ & 6 & 10 & 4 & 8 & 11 & 3 \\
\hline Diabetes mellitus no se cura & 7 & 12 & 5 & 8 & 12 & 4 \\
\hline $\begin{array}{l}\text { Existen dos tipos principales de diabe- } \\
\text { tes (1 y } 2 \text { ) }\end{array}$ & 4 & $12 *$ & 8 & 4 & 8 & 4 \\
\hline $\begin{array}{l}\text { El ingerir comida hace que se produzca } \\
\text { insulina }\end{array}$ & 6 & 5 & -1 & 6 & 5 & -1 \\
\hline $\begin{array}{l}\text { Es igual de importante la forma de } \\
\text { escoger los alimentos y su manera de } \\
\text { cocinarlos }\end{array}$ & 9 & 10 & 1 & 6 & 6 & 0 \\
\hline $\begin{array}{l}\text { Los alimentos de los diabéticos no son } \\
\text { diferentes a los de las demás personas }\end{array}$ & 3 & $11 *$ & 8 & 5 & 8 & 3 \\
\hline Total & 53 & $87^{*}$ & 34 & 59 & 67 & $8^{++}$ \\
\hline
\end{tabular}

CONTROL DE GLUCOSA

\begin{tabular}{|l|c|c|c|c|c|c|}
\hline $\begin{array}{l}\text { El ejercicio y los medicamentos no ele- } \\
\text { van la glucosa en sangre }\end{array}$ & 4 & 6 & 2 & 7 & 7 & 0 \\
\hline $\begin{array}{l}\text { 210 mg de glucosa en sangre es un } \\
\text { nivel alto }\end{array}$ & 10 & 11 & 1 & 7 & 12 & 5 \\
\hline $\begin{array}{l}\text { La valoración de glucosa en la orina no } \\
\text { es la mejor manera de hacerlo }\end{array}$ & 3 & 7 & 4 & 7 & 5 & -2 \\
\hline $\begin{array}{l}\text { Haciendo ejercicio regularmente se } \\
\text { necesita menos insulina }\end{array}$ & 3 & 8 & 5 & 5 & 6 & 1 \\
\hline $\begin{array}{l}\text { Los medicamentos no son más impor- } \\
\text { tantes que la dieta y el ejercicio }\end{array}$ & 9 & & & & & \\
\hline
\end{tabular}


Rafael Bustos, Blanca Aceves, Bertha del Toro, Patricia Martínez, Federico Pérez, Miguel Rodríguez, José Miguel Rolón, José de Jesús Vargas y Noelia Vargas

\begin{tabular}{|c|c|c|c|c|c|c|}
\hline CONOCIMIENTOS BASICOS & $\begin{array}{l}\text { RESPUESTA } \\
\text { INICIAL }\end{array}$ & $\begin{array}{l}\text { RESPUESTA } \\
\text { FINAL }\end{array}$ & $\begin{array}{l}\text { DIFEF } \\
\text { ENTR } \\
\text { PUES }\end{array}$ & $\begin{array}{l}\text { RES- } \\
\text { PUESTA } \\
\text { INICIAL } \\
\end{array}$ & \begin{tabular}{|l} 
RES- \\
PUESTA \\
FINAL \\
\end{tabular} & $\begin{array}{l}\text { DIFERENCIA } \\
\text { ENTRE RES- } \\
\text { PUESTAS }\end{array}$ \\
\hline $\begin{array}{l}\text { Presentar sudoración y temblores no } \\
\text { son síntomas de que subió la glucosa }\end{array}$ & 1 & 5 & 4 & 2 & 4 & 2 \\
\hline $\begin{array}{l}\text { El orinar seguido y tener sed no son } \\
\text { síntomas de que bajo la glucosa }\end{array}$ & 2 & 8 & 6 & 3 & 2 & -1 \\
\hline Total & 32 & $54^{*}$ & 22 & 38 & 43 & $5^{++}$ \\
\hline \multicolumn{7}{|l|}{ PREVENCION DE COMPLICACIONES } \\
\hline $\begin{array}{l}\text { Diabetes produce mala circulación de } \\
\text { la sangre }\end{array}$ & 7 & 11 & & 8 & 11 & 3 \\
\hline $\begin{array}{l}\text { Las heridas no cicatrizan más despacio } \\
\text { en el diabético }\end{array}$ & 5 & 11 & 6 & 6 & 9 & 3 \\
\hline $\begin{array}{l}\text { Los diabéticos deben de cuidarse al } \\
\text { cortarse sus uñas }\end{array}$ & 9 & 12 & 3 & 2 & $10 *$ & 8 \\
\hline $\begin{array}{l}\text { Las heridas en los diabéticos no deben } \\
\text { de curarse con alcohol y yodo }\end{array}$ & 2 & 5 & 3 & 0 & $4^{*}$ & 4 \\
\hline $\begin{array}{l}\text { Los riñones se pueden dañar por dia- } \\
\text { betes }\end{array}$ & 8 & 12 & 4 & 8 & 9 & 1 \\
\hline $\begin{array}{l}\text { Se puede presentar pérdida de sensibi- } \\
\text { lidad en pies y manos por diabetes }\end{array}$ & 6 & 12 & 6 & 6 & 12 & 6 \\
\hline $\begin{array}{l}\text { Los calcetines y medias elásticas no } \\
\text { son recomendados en los diabéticos }\end{array}$ & 4 & 11 & 7 & 5 & 7 & 2 \\
\hline Totales & 41 & $74^{*}$ & 33 & 35 & $62 *$ & 27 \\
\hline TOTAL GENERAL & 126 & $215^{*}$ & 89 & 132 & $172 *$ & $40^{++}$ \\
\hline
\end{tabular}

* Diferencia entre la evaluación inicial y final por cada grupo $\mathrm{p}<0.05$

${ }^{++}$Diferencia entre las diferencias de los dos grupos $\mathrm{p}<0.05$ 
Impacto de dos estrategias educativas en la adquisición de conocimientos sobre diabetes mellitus

Tabla 2 Diferencia en los promedios de respuestas adecuadas al inicio y final por cada una de las estrategias

\begin{tabular}{|l|l|l|l|l|l|l|}
\hline & \multicolumn{3}{|c|}{ GRUPO DE REFORZAMIENTO } & \multicolumn{3}{c|}{ GRUPO DE PERIODISMO } \\
\hline & $\begin{array}{l}\text { PROMEDIO } \\
\text { INICIAL }\end{array}$ & $\begin{array}{l}\text { PROMEDIO } \\
\text { FINAL } \\
\text { LROMEDIO DE } \\
\text { LAS DIFERENIAS } \\
\text { DE ANTESY DES- } \\
\text { PUES }\end{array}$ & $\begin{array}{l}\text { PROMEDIO } \\
\text { INICIAL }\end{array}$ & $\begin{array}{l}\text { PROMEDIO } \\
\text { FINAL }\end{array}$ & $\begin{array}{l}\text { PROMEDIO DE } \\
\text { LAS DIFEEN- } \\
\text { CIAS DE ANTESY } \\
\text { DESPUES }\end{array}$ \\
\hline $\begin{array}{l}\text { Conocimientos } \\
\text { basicos }\end{array}$ & $4.42 \pm 1.9$ & $7.25 \pm 1.4^{*}$ & $2.83 \pm 2.1$ & $4.92 \pm 2.3$ & $5.58 \pm 1.6$ & $0.67 \pm 1.2^{++}$ \\
\hline $\begin{array}{l}\text { Control de glu- } \\
\text { cemia }\end{array}$ & $2.67 \pm 1.5$ & $4.5 \pm 1.8^{*}$ & $1.83 \pm 1.6$ & $3.17 \pm 1.6$ & $3.58 \pm 1.7$ & $0.42 \pm 1.9^{++}$ \\
\hline $\begin{array}{l}\text { Prevencion de } \\
\text { complicaciones }\end{array}$ & $3.42 \pm 1.5$ & $6.18 \pm 0.83^{*}$ & $2.75 \pm 1.4$ & $2.92 \pm 1.6$ & $5.17 \pm 0.8^{*}$ & $2.25 \pm 1.4$ \\
\hline $\begin{array}{l}\text { Cuestionario en } \\
\text { general }\end{array}$ & $10.5 \pm 2.9$ & $17.92 \pm 3.4^{*}$ & $7.42 \pm 2.5$ & $11 \pm 4.04$ & $14.33 \pm 3.5^{*}$ & $3.33 \pm 3.3^{++}$ \\
\hline
\end{tabular}

* Diferencia entre la evaluación inicial y final por cada grupo $\mathrm{p}<0.05$

++ Diferencia entre las diferencias de los dos grupos $\mathrm{p}<0.05$

\section{Discusión}

El presente estudio se realizó aprovechando la organización de un Taller de Tenis que consistió en 15 sesiones de práctica de juego y 13 de trabajo del estudio de la evaluación de las estrategias educativas que se realizaron al mismo tiempo. Tanto los participantes como los padres de los mismos desconocían (previo a la inscripción en el Taller de Tenis) que se fuera a llevar a cabo esta actividad de aprendizaje paralela a las acciones deportivas, por lo que, para todos los participantes fue una sorpresa su realización.

Por otra parte, podemos considerar a la educación en salud como un recurso que no sólo afecta las cuestiones pedagógicas, sino también las sociales, económicas y políticas y que contempla la búsqueda participativa de las personas que se encuentran bajo el proceso de aprendizaje (Valadez et al., 2004). Visto desde esta perspectiva, la visión participativa del aprendizaje (Viniegra, 2008) en los adolescentes del estudio se pudo ver favorecida por varios aspectos tanto personales como familiares: a) la difusión nacional en medios de comunicación de factores de riesgo de enfermedades (principalmente por el impacto de la obesidad infantil en México), b) la experiencia que tuvieron los niños por haber convivido muchos de ellos con familiares diabéticos (padres, tíos o abuelos), y c) los mensajes en los medios de comunicación masiva de actividades de prevención masiva. Estas tres actividades de aprendizaje hicieron que los participantes presentaran en su gran mayoría interés en los temas y pudieran hacer significativos los conocimientos que se expresaron en las charlas (Villalón y Leclair, 2004). 
Rafael Bustos, Blanca Aceves, Bertha del Toro, Patricia Martínez, Federico Pérez, Miguel Rodríguez, José Miguel Rolón, José de Jesús Vargas y Noelia Vargas

Por otra parte, las pláticas se realizaron tratando de manejarlas con un lenguaje que fuera accesible a los participantes, contestando todas sus preguntas o dudas en forma inmediata, estas actividades de reforzamiento basado en la experiencia de los niños, tuvieron como resultado la comprensión en forma integral los mensajes de salud (Reyes, Grau y Labrada, 1999; Cervelló, 2000).

De antemano sabemos que un estímulo educativo producirá una respuesta de aprendizaje, la cual se puede reforzar de manera positiva o negativa provocando que la conducta se fortalezca o se debilite (Skinner, 1972). Se debe tener en consideración que la información durante el desarrollo de las ejecuciones, propiciará el grado de implicación del individuo, por ello, un requisito es que la calidad de esta sea significativa y además suficiente. Por otra parte, se requiere que los códigos lingüísticos sean reconocidos por los partícipes de la clase; con estos se pueden establecer los nexos deseables, entre lo que espera el educador y lo que entiende el participante. Los códigos demasiado elaborados o muy técnicos, dificultan la apropiación del mensaje por parte de los adolescentes (Valdivia, 2011).

El plan de trabajo de los adolescentes con reforzamiento en preguntas, durante el juego de tenis, presentó mejoría significativa en todos los campos estudiados durante el proyecto, la estrategia de competencia y retroalimentación inmediata a base de cuestionamientos sobre el tema, en conjunto con su experiencia y escasa cantidad de conocimientos previos hizo que los participantes tuvieran mayores conocimientos en forma significativa.

La realización de actividades de periodismo y elaboración de un periódico sobre diabetes mellitus, fue una estrategia aunada a las charlas que mejoraron el aprendizaje en forma general, y particularmente en las actividades de prevención de complicaciones. El periodismo actual se ha ido especializando cada vez más en áreas específicas del conocimiento. Una de estas áreas es la encaminada hacia temas de la salud. Sin embargo, a pesar de esto, la población continua estando mal informada, principalmente debido a: a) no es atractivo como noticia, b) no siempre es completa la información ofrecida, c) se transmiten ideas complejas que en ocasiones no son comprensibles (Ruiz, Iglesias y Ferrer, 2005), por lo que el acercamiento a esta técnica didáctica potencialmente hará que los adolescentes consulten con mayor frecuencia los periódicos para su aprendizaje, y no solamente como entretenimiento o información (Preciado, León y Fuentes, 2004).

El diseño realizado pudo haberse contaminado con el sesgo de obtención de información por parte de los participantes del estudio en forma independiente a los conocimientos adquiridos en las charlas. Para este concepto, se les solicitó a los participantes que no realizaran lecturas u obtención de conocimiento por otros medios agregados a las pláticas (al final declararon que ninguno lo realizó). El avance en el tiempo pudo hacer que los adolescentes de los dos grupos (principalmente los de periodismo) presentaran olvido de los conceptos de los primeros días de charla, por lo que fue poco su avance de aprendizaje. Pese a lo anterior, la presencia de familiares diabéticos (que fue similar en los dos grupos) pudo influir en la obtención de conocimientos por parte de la experiencia de ellos en su convivencia. Este aspecto fue muy interesante a tomar en cuenta sobre todo 
Impacto de dos estrategias educativas en la adquisición de conocimientos sobre diabetes mellitus

en el rubro de prevención de las complicaciones en los pacientes diabéticos, porque seguramente las experiencias de los familiares, que presentaron los adolescentes del estudio, lograron hacer que mejoraran sus conocimientos (en forma similar tanto en los participantes del reforzamiento como en los de periodismo). El resultado obtenido en el estudio presenta diferencias interesantes con otros realizados, en el aspecto de que los conocimientos sobre las complicaciones fueron mayores a los de conocimientos básicos o al de control de glucemia (UNESCO).

Los presentes resultados de conocimientos son parte del trabajo integral que se realizó con los participantes sobre el resultado de las estrategias educativas en diabetes mellitus. Para la evaluación de conocimientos adquiridos se manejó un instrumento, previamente utilizado por los autores del estudio, en población adulta sana y de diabéticos del sur de Jalisco, México (Bustos, 2007a \& Bustos et al., 2011). Es importante señalar que los conocimientos expresados en el inicio del trabajo en los adolescentes con respecto a los diabéticos y las personas sanas de la zona participantes en los otros estudios regionales son parecidos en el aspecto de conocimientos de la prevención de complicaciones.

La elaboración de estudios como el presente nos orilla a pensar en el establecimiento de modificaciones a los currículos nacionales e internacionales de la educación básica, a fin de garantizar

que la promoción de la salud, particularmente la alimentación correcta y el ejercicio físico constante y adecuado, sea un tema que si bien está presente actualmente, se refuerce y se aborde en más de una asignatura en todos los grados escolares (Secretaría de Educación Pública, 2010).

Debemos de enseñar a las personas (en este caso a los adolescentes) a que aprendan a tomar sus propias decisiones respecto a su salud. Los resultados satisfactorios encontrados en el trabajo, en el incremento de los niveles de conocimiento, demostraron la eficacia de la implantación de técnicas de aprendizaje incluso en actividades de juego independiente, tal como se ha reportado en otros estudios (Argote y Pérez, 2010; Rosen et al., 2006).

\section{Conclusión}

Las dos estrategias educativas utilizadas, hicieron que los alumnos presentaran un aumento de conocimiento en forma general. Sin embargo, la estrategia a base de reforzamiento de preguntas durante la práctica del juego de tenis presentó mejoría en todos los aspectos, superando incluso a los cambios que presentaron los de periodismo.

\section{Bibliografía}

Aguilera, L. L., G. M. Alonso, C. A. Castro, M. L. Cuadrado, O. M. Esparza, P. C. García et al. (2005). Protocolo de Salud Infantil, atención al niño sano. Madrid: MS Impresores.

Argote, O. F. y R. G. Pérez (2010). Promoción de salud familiar: intervención educativa en niños. Aten Fam, 17(4), 90-92. 
Rafael Bustos, Blanca Aceves, Bertha del Toro, Patricia Martínez, Federico Pérez, Miguel Rodríguez, José Miguel Rolón, José de Jesús Vargas y Noelia Vargas

Arredondo, A. (2011). "Costo de la Diabetes en América latina: Evidencias del caso mexicano". Value in Health, 14, 585-8.

Bustos, S. R. (2006). "Aprender a educar, una necesidad del Médico de familia en la atención de los pacientes diabético". Revista Paceña de Medicina Familiar, 3(3), 33-36.

—— H. G. López, N. E. Sánchez, P. R. Palomera, M. A. Barajas y G. J. Islas (2007a). “Conocimientos sobre diabetes mellitus en pacientes diabéticos tipo 2 tanto urbanos como rurales del occidente de México". Arch Med Fam, 9(3),150-4.

— , A. L. Florean, H. G. López, N. E. Sánchez, P. R. Palomera, M. A. Barajas, M. R. Bustos y M. A. Bustos (2007b). "Conocimientos usuales de los pacientes diabéticos en los estudiantes de Medicina del occidente de México". Educación Médica, 10(4), 225-32.

— , S. M. Ramírez, D. L. Orozco, C. M. Orozco, S. L. Valencia y C. M. Arias (2009). "Conocimientos básicos sobre influenza AH1N1 en los estudiantes universitarios del occidente de México". MPA-e Journal de Med Fam At Prim, 3(1-3) ,7-12.

— , S. R. Gildo, H. G. López, N. E. Sánchez, J. A. Peña y M. Pelayo (2011). "Conocimiento sobre su Enfermedad en Pacientes Diabéticos Tipo 2 Hospitalizados y de Consulta Externa del Occidente de México". MPA e Journal de Medicina Familiar y atención primaria Internacional, 5(2), 63-68.

— , M. A. Bustos, M. R. Bustos, A. I. Cabrera y F. J. Cortes (2011). "Falta de conocimiento como factor de riesgo para ser hospitalizados en pacientes diabéticos tipo 2". Arch Med Fam, $13(2), 62-71$.

Caldés, S., N. Cea, P. Crespo, V. Diez, A. Espino, S. Galán et al. (2005). "Una intervención educativa en niños de doce años en Madrid modifica sus conocimientos y habilidades de higiene bucodental?". Avances en Odontoestomatología; 21(3),149-157.

Cervelló, E. M. y F. J. Santos-Rosa (2000). "Motivación en las clases de educación física: un estudio de la perspectiva de las metas de logro en el contexto educativo". Revista de Psicología del Deporte, 9, 51-70.

Colomer-Revuelta, C., J. Colomer-Revuelta, R. Mercer, R. Peiró Pérez y L. Rajmil (2004). "La salud en la Infancia". Gac Sanit ,18(1), 39-46.

García, A. A., E. T. Villagomez, S. A. Brown, K. Kouzekanani y C. L. Hanis (2001). “The Starr County. Diabetes education study: Development of the Spanish-language Diabetes Knowledge Questionnaire". Diabetes Care, 24, 16-21.

García, M. L., C. González Elías y P. M. Aguilar (2010). "Intervención educativa en familiares de niños diabéticos". MEDICIEGO, 16 (suplemento 2).

Gómez, D. (2001). "La transición en epidemiología y Salud Pública ¿explicación o condena?". Revista Facultad de Salud Pública, 19(2), 57-74

Guibert, W. (1999). “Cómo hacer más efectiva la educación en salud en la atención primaria?”. Revista Cubana Med Gen Integr, 15(2), 176-83. 
Impacto de dos estrategias educativas en la adquisición de conocimientos sobre diabetes mellitus

International Diabetes Federation. Diabetes Atlas, 4th ed. International Diabetes Federation, 2009. Recuperado 10 de julio del 2013 desde http://www.idf.org/

International Diabetes Federation. Plan Mundial contra la diabetes 2011-2013. Recuperado el 10 de julio del 2013 desde http://www.idf.org/sites/default/files/attachments/GDP-Spanish.pdf

Kuri-Morales, A. (2011) "La transición en Salud y su impacto en la demanda de servicios". Gaceta Médica de México, 147, 451-4.

Lerman, I., A. López-Ponce, A. Villa, M. Escobedo, E. Caballero, M. Velasco et al. (2009). "Estudio piloto de dos estrategias para reforzar conductas de autocuidado y adherencia al tratamiento con pacientes de bajos recursos económicos con diabetes tipo 2". Gaceta Médica de México, 145(1), 15-19.

Ostegren, N. A., M. L. Caitlin, J. Pedersen, S. Skovlund y A. Greene (2007). "Necesidades y prioridades a la hora de mejorar el apoyo y la educación de los jóvenes: una llamada a la acción". Diabetes Voice, 52, 41-42.

Preciado, V., G. León y H. Fuentes. "Comunicación educativa en la prensa: suplementos infantiles en México”. Razón y Palabra. Diciembre 2003-enero 2004. Recuperado el 15 de julio del 2013 desde http://www.razonypalabra.org.mx/anteriores/n36/prensa.html

Pupo, A. L. y R. C. Carvajal (2009). "Estrategias metodológicas para la formación de las niñas y los niños como promotores de salud en las familias”. Revista Cubana de Salud Pública, 35(4), 139-53.

Reyes, W.G., J. A. Grau y M. C. Labrada (1999). “¿Cómo hacer más efectiva la educación en salud en la atención primaria?”. Revista Cubana Med Gen Integr, 15(2), 176-83.

Rosen, L., O. Manor, D. Engelhard, D. Brody, B. Rosen, H. Peleg et al. (2006). "Can a hand washing intervention make a difference? Result from a randomized controlled trial in Jerusalem preschools". Preventive Medicine, 42(1), 27-32.

Ruiz, M. L., S. M. Iglesias y A. J. Ferrer (2005). Prevención de la Obesidad y la Diabetes mellitus tipo 2. España, Mérida: Printex Artes Gráficas.

Secretaria de Educación Pública (2010). Acuerdo nacional para la salud alimentaria. Estrategias contra el sobrepeso y obesidad" (en línea). Programa de acción en el contexto escolar. México: Primera Edición Editorial Secretaría de Salud, pp. 9-21.

Skinner, B. (1972). Decisión educacional y evaluación. En Strom, R. Aprendizaje escolar y evaluación. Buenos Aires: Paidós

Valadez, F. I., F. M. Villaseñor y A. N. Alfaro (2004). "Educación para la salud: La importancia del concepto". Revista de Educación y Desarrollo, 1, 43-8.

Valdivia, J. (2011). "La retroalimentación correctiva y de reforzamiento en clases de educación física”. Trances, 3(5), 687-94.

Villalon, L. y C. A. Leclair (2004). "A participatory approach for the prevention of type 2 diabetes for francophone youth of New Brunswick”. Can J Diet Pract Res, 65(1), 15-21. 
Rafael Bustos, Blanca Aceves, Bertha del Toro, Patricia Martínez, Federico Pérez, Miguel Rodríguez, José Miguel Rolón, José de Jesús Vargas y Noelia Vargas

Viniegra Velázquez, L. (2008). "Hacia un nuevo paradigma en la educación”. Revista de Investigación Clínica 60(4), 337-55.

UNESCO. "Educación no formal". Recuperado el 25 de agosto del 2013 desde http://www.unesco. org/bpi/pdf/memobpi55_NFE_es.pdf

Recibido: 19/09/13

Dictaminado: 19/03/14

Corregido: 01/04/14

Aceptado: 09/04/14

\section{Esquema metodológico del estudio}

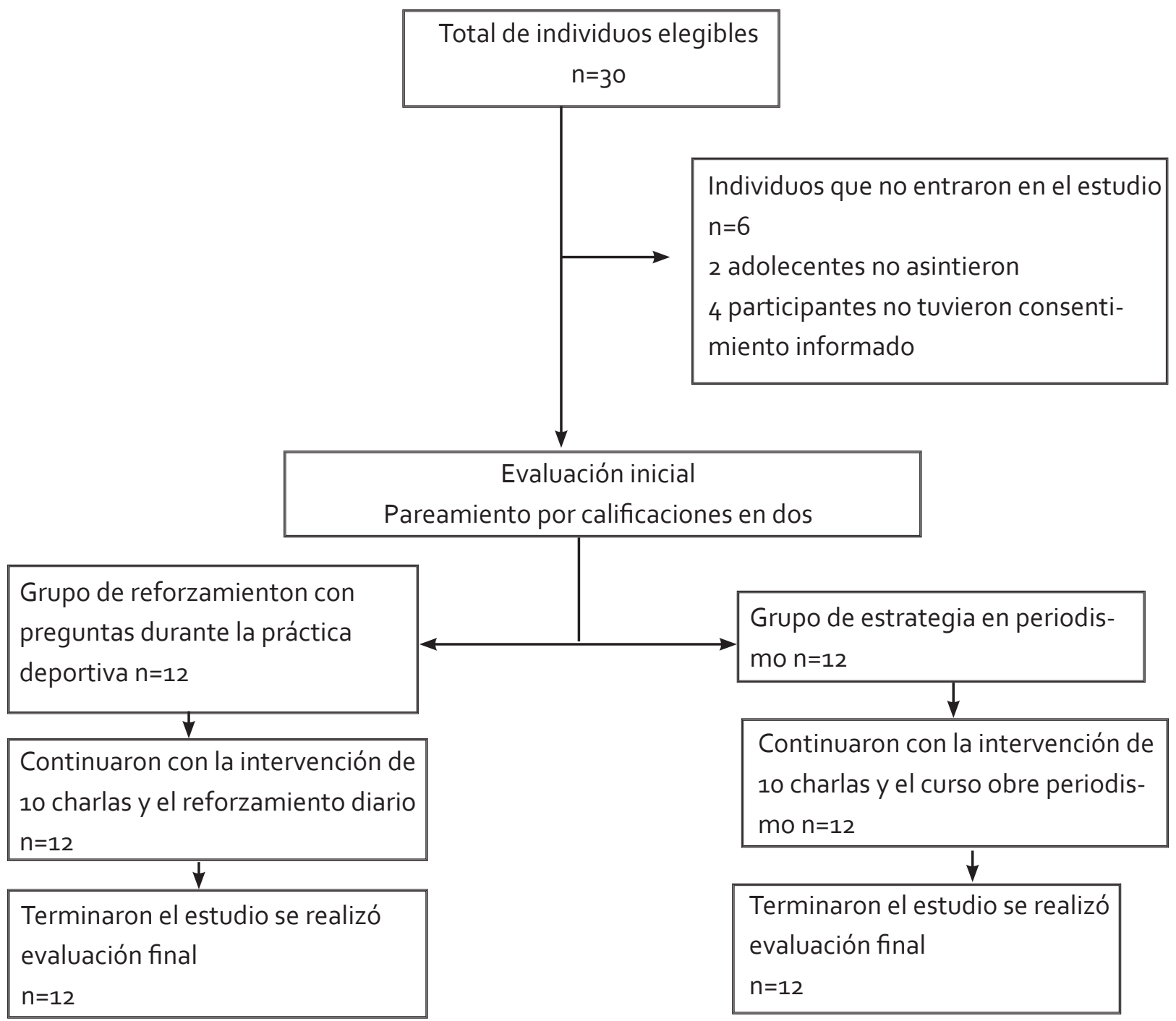

